\title{
Wireless Implantable Electronic Platform for Chronic Fluorescent-Based Biosensors
}

\author{
Pietro Valdastri*, Member, IEEE, Ekawahyu Susilo, Thilo Förster, Christof Strohhöfer, \\ Arianna Menciassi, Member, IEEE, and Paolo Dario, Fellow, IEEE
}

\begin{abstract}
The development of a long-term wireless implantable biosensor based on fluorescence intensity measurement poses a number of technical challenges, ranging from biocompatibility to sensor stability over time. One of these challenges is the design of a power efficient and miniaturized electronics, enabling the biosensor to move from bench testing to long term validation, up to its final application in human beings. In this spirit, we present a wireless programmable electronic platform for implantable chronic monitoring of fluorescent-based autonomous biosensors. This system is able to achieve extremely low power operation with bidirectional telemetry, based on the IEEE802.15.4-2003 protocol, thus enabling over three-year battery lifetime and wireless networking of multiple sensors. During the performance of single fluorescent-based sensor measurements, the circuit drives a laser diode, for sensor excitation, and acquires the amplified signals from four different photodetectors. In vitro functionality was preliminarily tested for both glucose and calcium monitoring, simply by changing the analyte-binding protein of the biosensor. Electronics performance was assessed in terms of timing, power consumption, tissue exposure to electromagnetic fields, and in vivo wireless connectivity. The final goal of the presented platform is to be integrated in a complete system for blood glucose level monitoring that may be implanted for at least one year under the skin of diabetic patients. Results reported in this paper may be applied to a wide variety of biosensors based on fluorescence intensity measurement.
\end{abstract}

Index Terms-Biotelemetry, calcium sensing, chronic implants, fluorescence-based biosensor, fluorescence resonant energy transfer (FRET), glucose monitoring, implantable devices.

\section{INTRODUCTION}

$\mathbf{R}$ ECENT achievements in miniaturized electronics and smart system integration have led to a proliferation of wearable and implantable devices for the monitoring of vital signs. These systems hold the promise to lower the threshold for early diagnosis and to dramatically enhance the quality of

Manuscript received September 22, 2010; revised November 27, 2010 and January 24, 2011; accepted February 20, 2011. Date of publication March 7, 2011; date of current version May 18, 2011. This work was supported by FP6 European Project P. Cezanne under Grant IST-2006-IP031867. Asterisk indicates corresponding author.

${ }^{*} \mathrm{P}$. Valdastri is with the BioRobotics Institute, Scuola Superiore Sant'Anna, 56127 Pisa, Italy (e-mail: pietro@sssup.it).

E. Susilo, A. Menciassi, and P. Dario are with the BioRobotics Institute, Scuola Superiore Sant'Anna, 56127 Pisa, Italy (e-mail: e.susilo@sssup.it; arianna@sssup.it; paolo.dario@sssup.it).

T. Förster and C. Strohhöfer are with Fraunhofer EMFT, 80686 Munich, Germany (e-mail: thilo.foerster@emft.fraunhofer.de; christof.strohhoefer@ emft.fraunhofer.de).

Color versions of one or more of the figures in this paper are available online at http://ieeexplore.ieee.org.

Digital Object Identifier 10.1109/TBME.2011.2123098 life, toward the ultimate implementation of personalized healthcare [1].

Pressure sensors embedded in disposable contact lenses [2] or in cardiovascular stents [3] are just two examples of how smart system integration may enable continuous monitoring of a physical parameter. Similarly, implantable chemical biosensors may have a disruptive impact in this field [4]. In particular, fluorescence techniques provide high sensitivity and ease of operation for chemical sensing, thus making fluorescence-based sensor commonly adopted worldwide [5]. A number of fluorescence microscopy and spectroscopy techniques based on the lifetime, anisotropy, or intensity of the emission of fluorescent probes have been developed over the years [6]. These enormously sensitive techniques are usually implemented in a lab-bench setting. The development of a portable, or even implantable, platform based on fluorescence intensity measurement could pave the way to a new generation of monitoring systems.

Among other chemical species that can be detected in this manner [7], [8], glucose is by far the most relevant one in diagnostics. The availability of a reliable long-term blood glucose level (BGL) sensor would have a disruptive impact on healthcare and diabetes management, considering that over $5 \%$ of the world's population suffers from diabetes [9] and this number is increasing rapidly [10]. Wireless electronic systems have been suggested for amperometric [11], [12] and near-infrared spectroscopy based [13] glucose sensors. However, problems with existing continuous BGL monitors have encouraged alternative approaches to glucose sensing [14]. In particular, solutions based on fluorescence intensity and lifetime have special advantages, including sensitivity and potential long-term stability. Special attention has been paid to the use of biosensors generating fluorescence intensity signals proportional to glucose level with the help of fluorescence resonant energy transfer (FRET) [15]-[17]. A transdermal FRET-based system for continuous BGL monitoring is reported in [18]. To further reduce invasiveness, functionalized fluorescent microparticles [19], [20] can be injected in the patient and used to transdermally monitor glucose concentration. Despite promising results, this latter approach is not self-contained, since it requires external light excitation and a video camera for image analysis.

In this spirit, a miniaturized implantable system for chronic fluorescence-based glucose sensing would allow continuous monitoring, without affecting the patient's daily lifestyle. Several challenges must be faced to achieve this relevant goal, most of which are related to the biosensor itself (e.g., biocompatibility and sensor stability over time). Concerning electronics, high energy excitation pulses must be provided to drive the sensor 


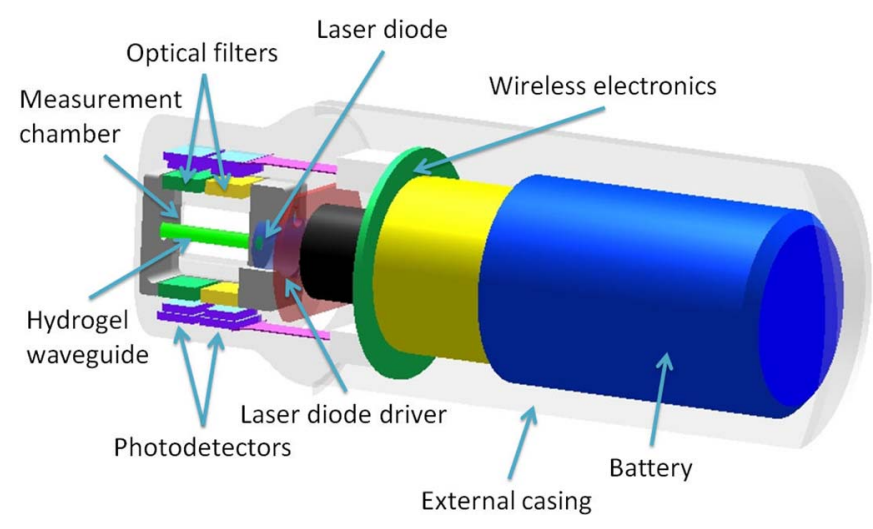

Fig. 1. Implant concept design.

and low noise photodetectors are required to collect the amount of energy transferred during the FRET event. Therefore, designing a low power, miniaturized and self-contained electronics is not straightforward. On the other hand, such electronics could easily be extended to the fluorescence-based detection of other chemical species, thus broadening its potential impact.

The aim of this paper, therefore, is to describe a miniaturized electronic platform for implanted long-term fluorescence biosensors. The platform allows fluorescence excitation and detection by driving a laser diode light source and phototransistors as detectors. Signals are amplified and transmitted across the skin to a personal mobile device, e.g., a cell phone or a laptop PC, where data can be displayed instantly. The electronic platform was designed bearing in mind the main features of implantable devices, namely small size, low power consumption, and safety. The functionality of the electronics was demonstrated in vitro with a FRET-based biosensor responding to changes in glucose concentration. In order to show how the proposed platform can be adapted to the monitoring of different chemical species, we repeated the in vitro experiment using a calcium-binding molecule. Finally, telemetry performance was assessed in terms of both safety and in vivo operation.

\section{SySteM OVERVIEW}

\section{A. Sensing Principle}

Despite the focus of this paper is on the electronics, an overview of the complete system is provided in this section, while a more detailed description is reported in [21]. The complete system is conceived to work as an under-skin implant, detecting analytes in interstitial fluids. It comprises a biosensor for signal generation, measurement, control and communication electronics, and a lithium intercalation cell as power supply. A schematic representation of the implant is reported in Fig. 1.

The biosensor is based on the principle of FRET [14], [22] between two fluorescent proteins, cyan fluorescent protein (CFP) and yellow fluorescent protein (YFP). These two proteins are covalently linked to an analyte binding protein (ABP). Analyte detection and transduction with this protein is schematically shown in Fig 2. Binding of the analyte to the bioreceptors induces a conformational change in the proteins and an incidental

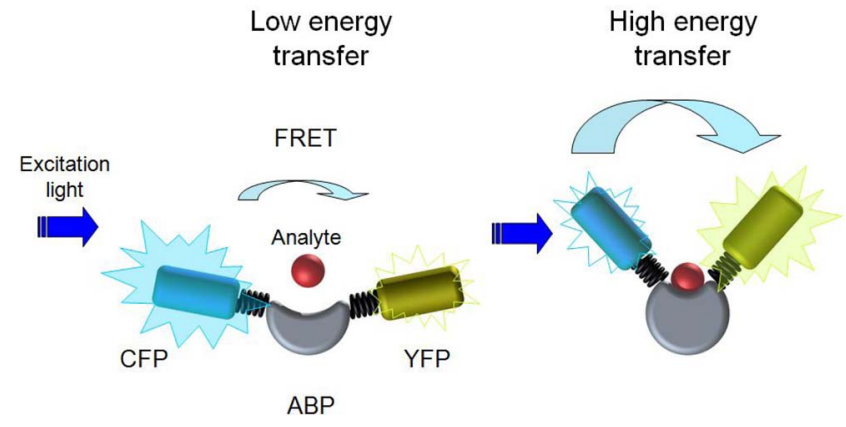

Fig. 2. (a) Schematic representation of analyte detection with the sensor protein. The fluorescent proteins CFP and YFP are covalently attached to the analyte recognition protein $\mathrm{ABP}$. (b) Upon analyte binding, $\mathrm{ABP}$ changes its conformation and alters the distance between the fluorescent proteins. The resulting change in fluorescence resonant energy transfer is proportional to the analyte concentration.

variation in the distance between the two fluorescent proteins. This translates into a change in intensity of the fluorescence emissions from the individual fluorescent proteins. The combined protein construct is immobilized inside a hydrogel by physical entrapment. The hydrogel has the shape of a cylindrical rod with diameter below $1 \mathrm{~mm}$ and length of around $8 \mathrm{~mm}$. It is suspended at its ends in a liquid-filled chamber, and fluorescence photodetectors are located at its sides. The hydrogel serves as optical waveguide for the fluorescence excitation light $(405 \mathrm{~nm})$ provided by a laser diode and coupled into the hydrogel through one of its end facets. Fluorescence is detected in two wavelength regions centered around $480 \mathrm{~nm}$ (CFP) and $527 \mathrm{~nm}$ (YFP). Wavelength discrimination is achieved by placing interference filters in front of the photodiodes. When the analyte concentration changes, the energy transfer between the fluorescent proteins changes accordingly, as also the relative intensity of fluorescence on the photodetectors. A schematic representation of the sensor head is included in Fig. 1. The sensor measures changes in analyte concentration inside the liquid-filled chamber, i.e., the measurement chamber. When the sensor is used in vivo or in liquids contaminated with larger molecules, bacteria or cells, access to the measurement chamber is closed by a membrane, which allows ions and glucose to freely diffuse into the sensor chamber while retaining cells and larger molecules.

As common for implanted biosensors, stability over time is the main hurdle to be faced with. As regards the described sensing strategy, the deactivation of ABP, CFP, and YFP, the decay of the florescence signal over time, pollution of the measurement chamber by serum proteins or blood clots, and the need for calibration are issues that need to be carefully addressed in future works. In the following, we focus on the design of an electronic platform enabling the implementation of this strategy. As the ones listed earlier, this is a relevant issue to be addressed, due to the severe specifications described in the next section.

\section{B. Requirements for Implant Electronics}

The in vivo repetitive sampling of a fluorescent-based biosensor poses several special requirements on the electronics, which we will address in the course of this article. 

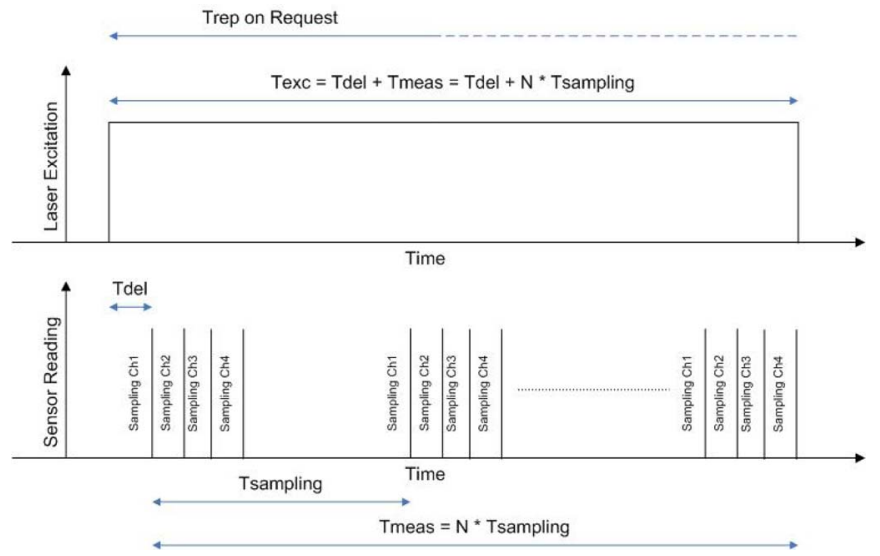

Fig. 3. Typical timing sequence for fluorescence-based sensor reading.

I/O features: The electronics must properly drive a laser light source and acquire light intensity from four different photodetectors. One pair of the photodetectors provides readings for the fluorescence intensities emitted from CFP and YFP, from which the FRET ratio is calculated. The second is a reference pair. It is worth mentioning that driving a laser light source, required for biosensor excitation, within a long-term wireless implant is extremely challenging in terms of power consumption and current requirements.

Timing: As highlighted in Fig. 3, each sensor reading is subject to specific timing requirements. First the laser diode must be switched on. Then, after a user adjustable delay time $T_{\text {del }}$ allowing stabilization of the signal, the sampling of the four photodetectors may start. Two other important parameters that the user may need to adjust are sampling time $T_{\text {sampling }}$ and number of samples to be acquired $N$. Once the measurement is over, the averages of the four signals over $N$ samples are made available to the end user. The last parameter is repetition time $\left(T_{\text {rep }}\right)$, which represents the user adjustable period for a single measurement.

Dimensions: The overall dimensions of the electronics must be as small as possible, thus minimizing discomfort once the sensor has been implanted under the skin. The target dimensions of the overall sensing system, including the measurement chamber, are $50 \mathrm{~mm}$ in length, and $20 \mathrm{~mm}$ in diameter, thus comparable to an implantable pacemaker.

Energy consumption: At least one year of operative battery lifetime is the minimum requirement to justify implantation. Once this time has elapsed, the implant may be replaced with a new one, in a day-hospital procedure.

Wireless connectivity: The wireless technology should guarantee data transfer reliability between the implant and a data collector located within a radius of a few meters from the patient. Bidirectional communication is preferable, in order to allow the user to interact with the implant and to adjust measurement parameters. Furthermore, by adopting a wireless technology that enables sensor networking, future scenarios with complex architectures may be envisaged (e.g., a single patient moving in a hospital environment and connecting to the closest data collector available for downloading sensor data, or a cluster of sensors, ei- ther implanted or worn by the patient, communicating together within a personal area network). Finally, the wireless energy density of telemetry passing through human tissues must comply with safety regulations [23] in order to prevent any possible risk for the patient.

\section{Architectural Overview of the Electronics}

In order to fulfill the aforementioned requirements, the system architecture needs to comprise a programmable device with an adequate amount of I/O, low power consumption, a compact package, and, possibly, bidirectional wireless communication features. This core component must drive the laser diode to provide excitation to the fluorescence biosensor and acquire the four properly amplified analog signals coming from the photodetectors. A switch driven by the programmable unit can be placed between the battery and the sensing circuitry. This would allow the analog circuitry to be switched off whenever possible, thus saving battery power.

A receiving unit-located outside the patient—-must be able to communicate with the implant, monitoring its status and enabling the end user to display and store the sensor readings and to share them with medical staff. The most straightforward way to achieve this goal is to develop a universal serial bus (USB) dongle that connects the implant to a laptop PC. This is the approach pursued in this article. Further development may integrate the receiving unit in a mobile device, e.g., a personal digital assistant or smart phone.

\section{Hardware Description}

An ideal candidate as core component of the implant electronics is the CC2430 wireless microcontroller from Texas Instruments. This device embeds an 8051 programmable microcontroller together with an IEEE 802.15.4-2003 [24] compatible transceiver in a $7 \mathrm{~mm} \times 7 \mathrm{~mm}$ package. An eight-channel $\Sigma \Delta$ analog to digital converter (ADC) provides 12-bit resolution sampling of the amplified photodetector signals. The CC2430 includes a five-channel direct memory access (DMA) controller, which can be used to relieve the microcontroller core of handling data movement operations and to achieve high overall performance with good power efficiency. In particular, the DMA controller can move data from a peripheral unit, such as the ADC, to memory with minimum CPU intervention. The on-board 16-bit timer can be used to obtain the desired $T_{\text {sampling }}$ during the acquisition of the $N$ photodetector samples. By exploiting the combined use of these three peripherals, a single sensor reading can be acquired minimizing microcontroller operations and consequently reducing power consumption. A 4-kB block of nonvolatile memory can be used to store the acquired data until receipt of an acknowledgment of correct transmission.

As regards telemetry, the CC2430 features a $2.4-\mathrm{GHz}$ transceiver complying with the IEEE 802.15.4-2003 standard [24] with a 250-kbps data rate. Despite tissue absorption at $2.4 \mathrm{GHz}$, previous work [25], [26] has demonstrated that this can still be a viable solution for implantable devices. A full ZigBee stack [27] can be implemented, when required, without any further hardware modification. Thanks to the availability 
of program flash up to $128 \mathrm{kB}$, the platform may also support application with a complex sensor network scenario. By using adjustable transmission power, the microcontroller can be programmed to always use the minimum level of energy required. This allows it to achieve reliable communication [26] and save battery. Few external components, e.g., a 32-MHz crystal and a surface mount balun (BD2425N50200A00, Anaren) for antenna impedance matching, are required for the correct operation of the radio section. In the present application, a 31-mm long wire serves as $\lambda / 4$ whip antenna.

Four different power modes can be used for the CC2430 to manage power consumption. In particular, a deep sleep mode, featuring nearly zero power consumption, can be used until the system is implanted under skin. An external magnetic switch can be used to wake up the microcontroller from the deep sleep mode. Once the implant is in place, the microcontroller can alternate operation between full functional mode and sleep mode, as explained in Section II-E. A watchdog timer is also provided, thus preventing the implant from code failure.

Other features of the CC2430 used in this application include temperature and battery level monitoring. Whenever the device is implanted and activated, the built-in temperature sensor can monitor the patient's body temperature, while the battery level monitor can provide real time information on the state of the power source and report to the central station when approaching a critical level.

The analog front end is responsible for the conversion of the fluorescence signals from CFP and YFP to a voltage that is passed on to the wireless microcontroller. As first step, the photocurrent generated by the fluorescence of the FRET proteins is converted to a voltage with a conversion factor of $10^{6} \mathrm{~V} / \mathrm{A}$. This is achieved with the help of a first operational amplifier (AD8034, Analog Devices) stage applied to the photodetector (TEMT6000, Vishay). As second step, the voltage undergoes further amplification in a differential amplifier. A reference voltage supply (ADR361, Analog Devices) included in the analog front end helps stabilize the output voltage and minimize the offset during the second stage amplification.

Two identical photodetectors are combined into a single unit, sharing common voltage supply, ground and reference. However at the signal conditioning stage, each of them has its own independent circuit. Output voltages from these circuits are proportional to the photocurrents and can be fed directly to the microcontroller ADC inputs, necessitating a mere four connections between the main control board and the analog front end.

A blue-violet (405 nm) laser diode (DL-4146-101S, Sanyo) is used to excite the fluorescent proteins. A dedicated laser diode driver (iC-WJ, iC-Haus) allows for power output regulation, providing a simple on/off interface for the microcontroller.

Both the analog front end and the laser diode driving circuitry require $5.5 \mathrm{~V}$ as power supply, while the selected battery (developed for this specific application by Tadiran Batteries in a 1520 package, providing $360 \mathrm{mAh}$ and supplying current spikes up to $750 \mathrm{~mA}$ ) operates at $4 \mathrm{~V}$. To match the two voltage levels, a stepup converter (MAX643, Maxim Integrated Circuits) is used in the adjustable-output configuration, thus requiring two additional external resistors. The oscillation frequency of the stepup
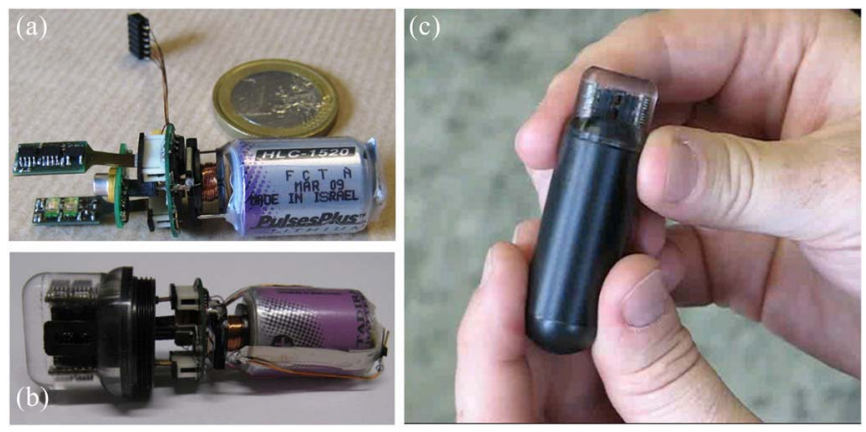

Fig. 4. (a) Miniaturized electronics. (b) Sensor device before encapsulation. (c) Final packaging of the sensor.

is $37 \mathrm{kHz}$. In order to provide enough current to the laser diode after voltage conversion, the stepup is operated in a high output current mode. This requires a $10.4 \mathrm{~mm} \times 9.4 \mathrm{~mm} \times 5.8 \mathrm{~mm}$ surface mount power inductor (B82475A1, Epcos), a highspeed switching $N$-channel enhancement mode MOS transistor (BSH103, Philips Semiconductors) and a fast switching diode (BAR64-04, Siemens). Finally, an analog switch (MAX4736, Maxim Integrated Circuits) is integrated that allows permanent or temporal disconnection of all the aforementioned analog parts by the microcontroller, in order to save power.

Three different boards were designed to accommodate all the components. The main control board hosts the wireless microcontroller, the analog switch, the stepup converter and the connectors to the battery and to the other boards. The board is circular with 17-mm diameter and is built upon four layers in rigid flexible circuit material reaching a total thickness of $12.3 \mathrm{~mm}$ after the components have been mounted on the top and bottom layers. Regarding layer functionality, all the layers are used as signal planes. Only a small area on the third layer is left out as a ground plane in compliance with the Anaren application note [28]. This ground plane is recommended for correctly matching the impedance of the balun to the whip antenna.

A second board comprises the laser diode and its driver, and plugs into the main board by a two-pole connector. The board measures $13 \mathrm{~mm}$ in diameter and $4 \mathrm{~mm}$ in thickness (with mounted components) and is shaped to fit into the bottom of the measurement chamber.

The analog front end is fabricated on a double-sided rigid-flex printed circuit board measuring $10.5 \mathrm{~mm} \times 5.5 \mathrm{~mm}$. Two front end boards, implementing two photodetectors each, are connected to the main control board by means of custom designed four-pole flexible circuit connectors (CviLux Corporation).

The completely assembled electronics is represented in Fig. 4(a). The two analog front end boards and the laser diode board are designed to be first integrated and packaged with the measurement chamber, and then connected to the main board, as shown in Fig. 4(b). As final step of system integration, a polyarylether ether ketone can be screwed on the bottom of the measurement chamber, thus sealing the implant [Fig. 4(c)]. The final dimensions are $63 \mathrm{~mm}$ in length and $21 \mathrm{~mm}$ in diameter.

As regards the external unit, a CC2430 is interfaced to a serial-USB signal converter (FT232R, FTDI Chip) through the 
asynchronous serial port (UART). This dongle is connected to the USB port of a standard PC, where a bespoke user interface, developed in Labview 8.2 (National Instruments, Austin, TX), is installed.

\section{E. Code Description}

Regarding firmware, the architecture comprises three different programmable units: the first unit is the $\mathrm{CC} 2430$ integrated in the implant. It communicates with the CC2430 of the USB dongle, which is the second programmable unit. The firmware codes for both these units were developed and debugged by using IAR Embedded Workbench (IAR Systems). The third programmable unit is the PC, where the user interface is installed. As a result, three independent codes running in parallel, but interacting with each other, were developed.

Since the platform needs to be able to execute different commands issued by the user (e.g., fluorescent-based sensor reading, temperature measurement, battery level enquiry, adjustment of measurement parameters, and setting of transmission power level), each command is codified with an ASCII symbol. Similarly, all the possible system failures that may occur within the operation (e.g., implant out of communication range, USB dongle failure) are properly codified and presented on the user interface.

During the code development phase, top priority was given to the implant firmware, especially to minimizing power consumption and to maximizing battery lifetime. For this reason, the implant is programmed to cyclically wake up every $T_{\text {sleep }}$ and to request a command from the external unit. If the USB dongle is within communication range and the user has already issued a command, the implant receives it and acts accordingly. Once the command has been executed, the implant goes back to sleep mode. If the USB dongle is not nearby or the user has not issued any command within a $T_{\text {timeout }}$ (set to $25 \mathrm{~ms}$ in the present application), the implant goes back to sleep mode as well. $T_{\text {sleep }}$ can be adjusted by the user by making a tradeoff between responsiveness of the implant and its battery lifetime.

The USB dongle code and the user interface are built around the implant code, allowing for a reliable communication and an effective failure management. The user interface allows the user to select the identification number of a specific implant, to set all the different parameters of a single measurement (i.e., $T_{\mathrm{del}}$, $T_{\text {sampling }}, N, T_{\text {rep }}$ ), to visualize and store the measurements, and to gather temperature and battery voltage. The received signal strength may also be displayed thanks to the receive signal strength indicator CC2430 feature.

\section{EXPERIMENTAL RESULTS}

\section{A. In Vitro Functionality Test}

Preliminary functionality tests were carried out with the electronic platform and a hydrogel waveguide biosensor [21] containing glucose binding proteins [29], [30] in the FRET compound molecule.

Tests were performed by dipping the hydrogel waveguide sensor into a solution mimicking human interstitial fluid. It consists

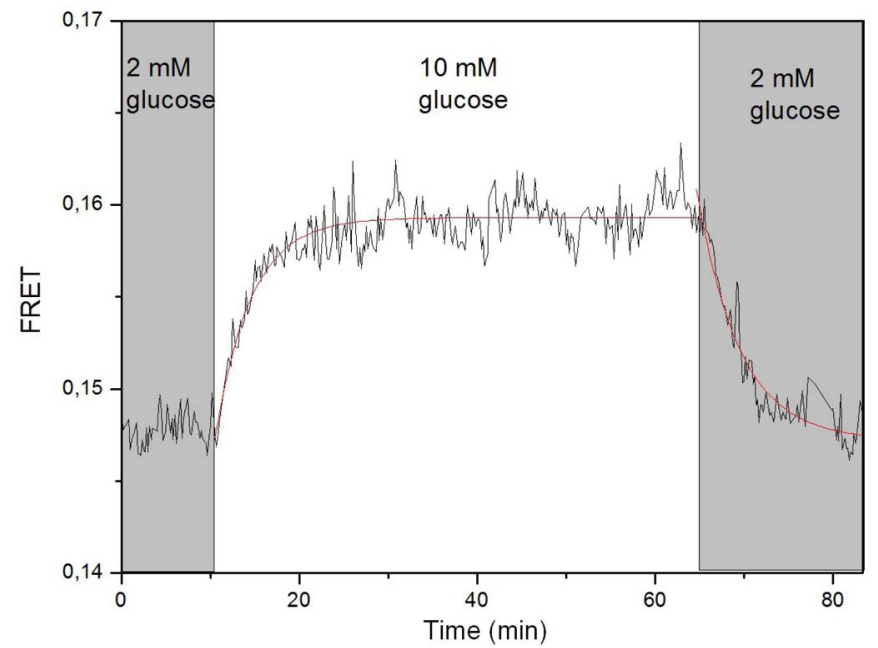

Fig. 5. FRET response to glucose binding and release, obtained from the fluorescence signals measured by the sensor. Measurement parameters: $N=$ $256, T_{\text {del }}=255 \mathrm{~ms}, T_{\text {sampling }}=100 \mu \mathrm{s}, T_{\mathrm{rep}}=10 \mathrm{~s}$.

of 10-mM HEPES buffer solution adjusted to $\mathrm{pH} 7.4$ with $\mathrm{HCl}$, and containing 122-mM NaCl, 25-mM NaHCO $3,3-\mathrm{mM} \mathrm{KCl}$, $1.2-\mathrm{mM} \mathrm{CaCl}_{2}, 1.2-\mathrm{mM} \mathrm{MgCl}_{2}, 0.5-\mathrm{mM} \mathrm{K}_{2} \mathrm{HPO}_{4}$. A $30-\mathrm{mg} / \mathrm{ml}$ bovine serum albumin was added to simulate the protein content in interstitial fluid. The sensor was placed repeatedly in these solutions containing $2-\mathrm{mM}$ and $10-\mathrm{mM}$ glucose, to evaluate the sensor response. All experiments were performed at $37^{\circ} \mathrm{C}$. Measurements were performed every $10 \mathrm{~s}$, adopting the following parameters: $N=256, T_{\text {del }}=255 \mathrm{~ms}, T_{\text {sampling }}=100 \mu \mathrm{s}$.

A typical measurement is shown in Fig. 5. The FRET signal was calculated as the ratio between the fluorescence emission from YFP and CFP. Fig. 5 shows an initially stable signal at 2 -mM glucose concentration. At time $t=10 \mathrm{~min}$, the sensor was immersed in 10-mM glucose solution. After a transitory period of roughly $10 \mathrm{~min}$, the signal stabilizes at a higher FRET level. The signal returns to the lower level when the sensor is placed back into the solution with low glucose concentration. The transition between low and high FRET levels and vice versa is caused by glucose diffusion into and out of the sensor. It shows exponential behavior (drawn line in Fig. 5) with a time constant of around 4-5 min, which is determined by the analyte's diffusion into the hydrogel waveguide.

Key to the detection of glucose is the particular FRET protein employed in the hydrogel waveguide. By simply replacing that with a similar one sensitive to a different analyte, other vital parameters may be monitored with the same electronic platform. To prove this, we replaced the hydrogel used for the previous test with a similar one, containing a calcium sensitive FRET compound.

Similar tests were performed by dipping the hydrogel waveguide sensor into 50-mM TRIS buffer solutions adjusted to $\mathrm{pH} 8$ with $\mathrm{HCl}$, and containing either 0 - or $5-\mathrm{mM} \mathrm{CaCl}_{2}$. Initially, the sensor was placed into a buffer solution without $\mathrm{CaCl}_{2}$ for $15 \mathrm{~min}$, then for $15 \mathrm{~min}$ into a buffer solution containing $5-\mathrm{mM}$ $\mathrm{CaCl}$, and finally once again into the solution without $\mathrm{CaCl}_{2}$. Measurements were performed every $10 \mathrm{~s}$, adopting the same 


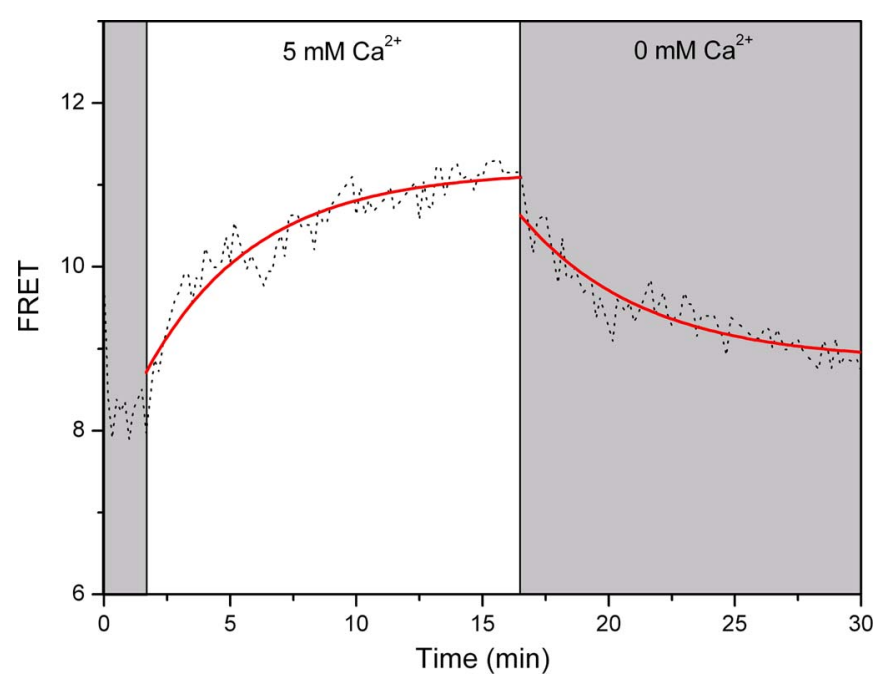

Fig. 6. FRET response to calcium binding and release, obtained from the fluorescence signals measured by the sensor. Measurement parameters: $N=$ $256, T_{\mathrm{del}}=255 \mathrm{~ms}, T_{\mathrm{sampling}}=100 \mu \mathrm{s}, T_{\mathrm{rep}}=10 \mathrm{~s}$.

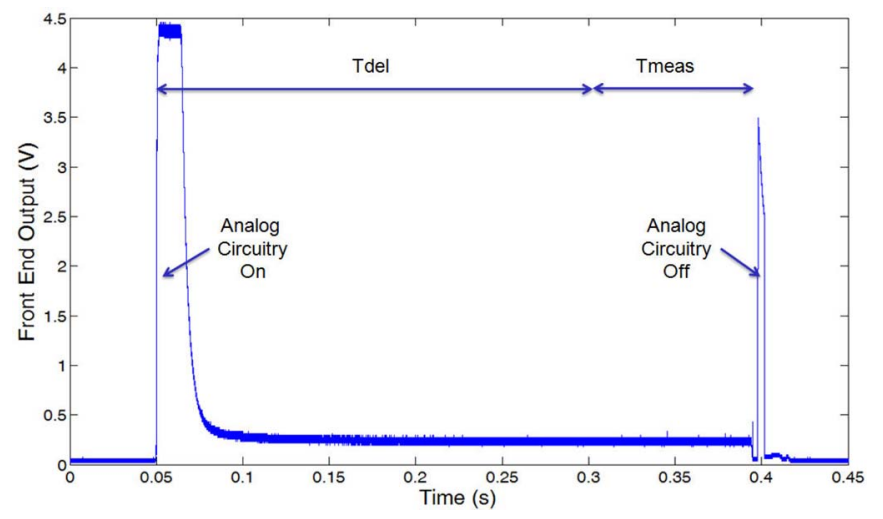

Fig. 7. Typical voltage output from a single channel of the analog front end for the following parameters setting: $N=256, T_{\mathrm{del}}=255 \mathrm{~ms}, T_{\text {sampling }}=$ $100 \mu \mathrm{s}$.

measurement parameters as aforementioned. A typical measurement is shown in Fig. 6, demonstrating similar performance of calcium sensing as demonstrated in Fig. 5 for glucose sensing.

These results demonstrate the electronic platform's potential beyond in vivo sensing, in applications such as distributed autonomous environmental monitoring, where it can serve as the sensor control and communications unit of a sensor network.

\section{B. Timing}

A plot showing the typical voltage output from a channel of the analog front end during a single measurement is reported in Fig. 7. As previously explained, the microcontroller starts sampling the voltage $T_{\text {del }}$ after the laser diode has been switched on. $N$ samples are acquired, each every $T_{\text {sampling, }}$ and then averaged to provide a single value per channel. The delay time $T_{\text {del }}$ should therefore be chosen so that the sampling starts only once the photodetector voltage has reached its steady state value. On the other end, $T_{\text {del }}$ should be minimized in order to save

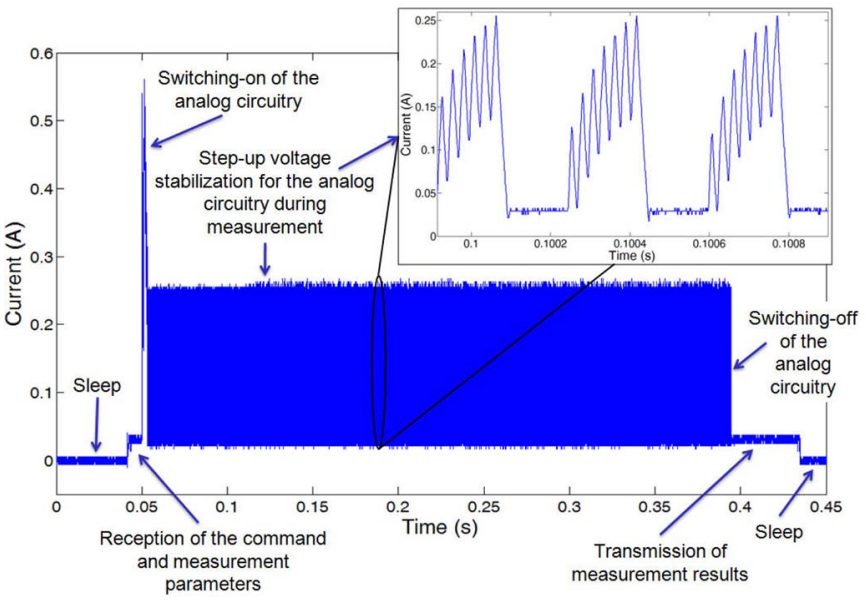

Fig. 8. Current consumption for the following parameters setting: $N=256$, $T_{\text {del }}=255 \mathrm{~ms}, T_{\text {sampling }}=100 \mu \mathrm{s}$.

energy. $N$ and $T_{\text {sampling }}$ must be adjusted considering the noise of the voltage signal during the measurement time. With the help of a plot as the one shown in Fig. 7, the measurement settings can be optimized. In particular, Fig. 7 shows the signal pulse from an in vitro glucose test with the fluorescence sensor. The mean value of the voltage output reaches $98 \%$ of its regime after $81 \mathrm{~ms}$; therefore, $T_{\text {del }}$ can be reduced by about $70 \%$ to increase battery lifetime.

\section{Power Consumption}

In order to quantify the current consumption of the complete electronic platform, the voltage drop across a $1-\Omega$ resistor placed in series to the positive voltage supply terminal was acquired during a single measurement of the biosensor. This measurement was performed with a 2-GS/s digital storage oscilloscope (TPS2024, Tektronix, Inc.). The plot shown in Fig. 8 shows the single phases of the measurement and quantifies each single contribution in terms of power consumption. In particular, the implant exits from sleep mode and requests a command. In this case, the request for a single biosensor measurement and the related parameter setting $\left(N=256, T_{\text {del }}=255 \mathrm{~ms}\right.$, $T_{\text {sampling }}=100 \mu \mathrm{s}$ ) are received. Then, the analog circuitry, including the laser diode, is switched on for $T_{\text {exc }}$, as defined in Fig. 3 and the measurement is performed. Directly following the measurement, the averaged 12-bit values of the four amplified photodetector signals are transmitted to the external unit and the implant goes back to sleep mode. It is worth mentioning that the periodic peaks and valleys of current reported in the inset of Fig. 8 are due to the stepup voltage converter, working at $37 \mathrm{kHz}$. These peaks show a periodic current drain, required to hold the voltage level to $5.5 \mathrm{~V}$, starting from $4 \mathrm{~V}$.

As detailed in [26], battery lifetime can be estimated by using the following average current consumption:

$$
\bar{I}=\frac{\sum_{i} I_{i} T_{i}}{\sum_{i} T_{i}}
$$

where $I_{i}$ and $T_{i}$ are the current consumption and the time interval related to a single action performed by the system within 
a periodic operation. Given the parameter setting used, a single sensor measurement requires an $\bar{I}_{\text {sensing }}=94 \mathrm{~mA}$ for a total time of $T_{\text {sensing }}=392 \mathrm{~ms}$. In the case, of chronic BGL monitoring, clinical considerations require that a single measurement should be acquired every $30 \mathrm{~min}$. Therefore, $T_{\text {sleep }}=$ $1799.6 \mathrm{~s}$ may be assumed. As regards $I_{\text {sleep }}$, since this is below the resolution of the test bench equipment used, the value was estimated from the datasheet of the components used as $I_{\text {sleep }}=$ $1.5 \mu \mathrm{A}$. By applying (1), an average current consumption of $\bar{I}=$ $22 \mu \mathrm{A}$ is obtained, which translates into over 22 months of continuous operation using the previously mentioned battery with $360-m A h$ capacity. A duration of 40 months can be achieved by optimizing $T_{\mathrm{del}}$ as reported earlier.

\section{Tissue Exposure to Electromagnetic Fields}

Once the described electronics demonstrated that it could effectively drive a fluorescence-based sensor for sufficient time to justify implantation, further tests were performed to assess the telemetric link.

The maximum value of plane wave power density emitted by the implantable unit was measured in order to compare it with international safety regulations. The power density was measured by a portable field strength meter (8053, PMM, Italy) located in the closest proximity to the module antenna. Its probe was oriented along the maximum radiation of the antenna, resulting in $0.002 \mathrm{~W} / \mathrm{m}^{2}$. This value is considerably lower than the ICNIRP reference level for general public exposure to timevarying electric and magnetic fields [23], fixed at $10 \mathrm{~W} / \mathrm{m}^{2}$ for a signal frequency of $2.4 \mathrm{GHz}$. Thus, the implant can be considered safe in electromagnetic terms.

\section{E. Wireless Connectivity}

Finally, the system was implanted under the skin of a pig in order to assess telemetry performances in an in vivo scenario. The experiment was performed in an authorized laboratory, with the assistance and collaboration of a specially trained medical team, in accordance to all ethical considerations and regulatory issues related to animal experiments. A $35-\mathrm{kg}$ domestic swine was sedated with a Ketamine injection in addition to Propofol and Stresnyl dosed intravenously. After intubation, anesthesia was maintained with Enflurane. The sensor, in its final packaging configuration, was implanted under the skin (3-cm depth) in the pelvic region of the swine [see Fig. 9(a)] and the incision was sutured. The USB dongle-shown in close proximity to the implant in Fig. 9(b)—was placed $2 \mathrm{~m}$ from the animal, in the operating room environment. A temperature sensor reading request was issued by the user interface every $10 \mathrm{~s}$ for a total of $4 \mathrm{~h}$. At the end of the experiment over $98 \%$ of telemetric transmissions of the temperature reading were successfully achieved. The $2 \%$ loss may be due to electromagnetic interferences from other instrumentations used for measuring physiological parameters such as electrocardiogram and pressure. These failures do not represent a critical issue in an operative scenario, because a second sensor reading can be requested by the external unit should the first one fail.

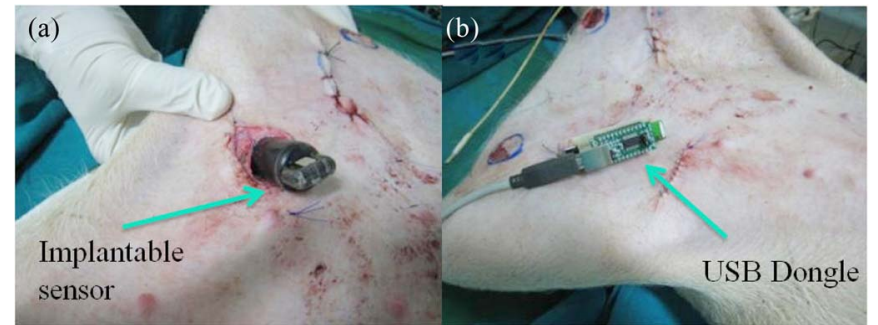

Fig. 9. In vivo test of telemetry. (a) Sensor before under-skin implantation. (b) USB dongle placed in proximity to the implanted sensor.

\section{CONCLUSION}

The main goal of this paper was to describe a miniaturized wireless electronic platform, suitable for in vivo monitoring of chemical species, such as glucose or calcium. Our solution enables long-term wireless monitoring of an implanted fluorescent-based sensor in a miniaturized package, measuring $63 \mathrm{~mm}$ in length and $21 \mathrm{~mm}$ in diameter. Over three years, operational battery lifetime can be obtained by properly adjusting measurement parameters. The proposed electronic platform can be easily applied to the monitoring of a different analyte by changing the binding protein embedded in the FRET compound molecule, as demonstrated by in vitro tests performed first with a glucose sensitive FRET compound, and then with a calcium sensitive one. Wireless networking of multiple sensors can be implemented without any further hardware modification, thanks to a IEEE802.15.4-2003 protocol compliant technology. Further tests demonstrated the safety of the platform as regards exposure to time-varying electric and magnetic fields and the functionality of the telemetric link once the system was implanted under skin.

Despite these encouraging results, some current limitations to the study described here require comments. As a first important step, the complete system needs to be validated by long-term in vitro and in vivo trials in order to assess sensor stability over time, with particular emphasis on both sensor packaging and the interface of the sensor with interstitial fluids. While a purposely developed membrane may shield the measurement chamber from pollutant, calibration will likely involve referencing from time to time with fingerprick measurements, as well as the use of an internal calibrant, which mimics the drift of the glucose sensitive protein. Thanks to bidirectional wireless communication, possible adjustments of sensor calibration can be implemented straightforwardly, thus preventing the need of explanting the system.

Another direction of future work will focus on further miniaturization, in order to meet the specified dimensional requirements. Reduction in volume can be achieved by optimizing the arrangement of the internal electronic components or by adopting a smaller battery that would still provide one year lifetime. Finally, despite performing preliminary safety validation, all the steps for clinical approval must be taken before applying the platform to humans.

To conclude, it is worth mentioning that all these further steps will originate from the electronic platform described in this 
paper, which represents a valid reference for any long-term implantable wireless system exploiting a fluorescence-based sensing principle.

\section{ACKNOWLEDGMENT}

The authors would like to thank T. Economou (IMBBFORTH, Iraklion, Greece) for providing the sensing proteins, I. Lacik (Polymer Institute of the Slovak Academy of Sciences, Bratislava, Slovakia) for the hydrogel waveguides, E. Elster from Tadiran Batteries, Kiryat Ekron, Israel, for providing the power source, and R. Di Leonardo and R. Lazzarini for their invaluable help.

\section{REFERENCES}

[1] K. Malasri and L. Wang, "Securing wireless implantable devices for healthcare: Ideas and challenges," IEEE Commun. Mag., vol. 47, no. 7, pp. 74-80, Jul. 2009.

[2] M. Leonardi, E. M. Pitchon, A. Bertsch, P. Renaud, and A. Mermoud, "Wireless contact lens sensor for intraocular pressure monitoring: Assessment on enucleated pig eyes," Acta Ophthalmologica, vol. 87, no. 4, pp. 433-437, 2009.

[3] E. Y. Chow, A. L. Chlebowski, S. Chakraborty, W. J. Chappell, and P. P. Irazoqui, "Fully wireless implantable cardiovascular pressure monitor integrated with a medical stent," IEEE Trans. Bio.-Med. Eng., vol. 57, no. 6, pp. 1487-1496, Jun. 2010.

[4] P. Tathireddy, L. Rieth, A. Sharma, and F. Solzbachert, "Implantable microsystems and neuro electronic interfaces," in Bionic Health: Next Generation Implants, Prosthetics and Devices, London, U.K.: IET, 2009, pp. 1-22.

[5] L. Basabe-Desmonts, D. N. Reinhoudt, and M. Crego-Calama, "Design of fluorescent materials for chemical sensing," Chem. Soc. Rev., vol. 36, pp. $993-1017,2007$.

[6] J. R. Lakowicz, Topics in Fluorescence Spectroscopy. New York: Springer-Verlag, 1994.

[7] J. R. Siqueira, Jr., L. Caseli, F. N. Crespilho, V. Zucolotto, and O. N. Oliveira, Jr., "Immobilization of biomolecules on nanostructured films for biosensing," Biosens. Bioelectron., vol. 25, no. 6, pp. 1254-1263, 2010.

[8] C. R. Taitt, G. P. Anderson, and F. S. Ligler, "Evanescent wave fluorescence biosensors," Biosens. Bioelectron., vol. 20, no. 12, pp. 2470-2487, 2005.

[9] G. A. Urban, "Micro-and nanobiosensors-state of the art and trends," Meas. Sci. Technol., vol. 20, no. 1, p. 012001, 2009.

[10] S. Wild, G. Roglic, A. Green, R. Sicree, and H. King, "Global prevalence of diabetes," Diabetes Care, vol. 27, no. 5, pp. 1047-1053, May 2004.

[11] R. D. Beach, R. W. Conlan, M. C. Godwin, and F. Moussy, "Towards a miniature implantable in vivo telemetry monitoring system dynamically configurable as a potentiostat or galvanostat for two- and three-electrode biosensors," IEEE Trans. Instrum. Meas., vol. 54, no. 1, pp. 61-72, Feb. 2005.

[12] M. M. Ahmadi and G. A. Jullien, "A wireless-implantable microsystem for continuous blood glucose monitoring," IEEE Trans. Biomed. Circ. Sys., vol. 3, no. 3, pp. 169-180, Jun. 2009.

[13] K. S. Kanukurthy, M. B. Cover, and D. R. Andersen, "Data acquisition unit for an implantable multi-channel optical glucose sensor," in Proc. IEEE Electro. Inform. Technol. (EIT), 2007, pp. 32-37.

[14] J. C. Pickup, F. Hussain, N. D. Evans, O. J. Rolinski, and D. J. Birch, "Fluorescence-based glucose sensors," Biosens. Bioelectron., vol. 20, no. 12 , pp. $2555-2565,2005$.

[15] B. Tang, L. Cao, K. Xu, L. Zhuo, J. Ge, Q. Li, and L. Yu, "A new nanobiosensor for glucose with high sensitivity and selectivity in serum based on fluorescence resonance energy transfer (FRET) between CdTe quantum dots and Au nanoparticles," Chem.-Eur. J., vol. 14, no. 12, pp. 3637-3644, 2008.

[16] F. Khan, L. Gnudi, and J. C. Pickup, "Fluorescence-based sensing of glucose using engineered glucose/galactose-binding protein: A comparison of fluorescence resonance energy transfer and environmentally sensitive dye labelling strategies," Biochem. Biophys. Res. Commun., vol. 365, no. 1, pp. 102-106, 2008.

[17] A. Bartolome, L. Smalls-Mantey, D. Lin, G. Rao, and L. Tolosa, "Fretbased glucose monitoring for bioprocessing," in Genetically Engineered

Probes for Biomedical Applications, vol. 6098, no. 1. San Jose, CA: SPIE, 2006, p. 60980L.

[18] R. Ballerstadt, C. Evans, A. Gowda, and R. McNichols, "In vivo performance evaluation of a transdermal near-infrared fluorescence resonance energy transfer affinity sensor for continuous glucose monitoring," Diabetes Technol. Therapeut., vol. 8, no. 3, pp. 296-311, 2006.

[19] H. Shibata, Y. J. Heo, T. Okitsu, Y. Matsunaga, T. Kawanishi, and S. Takeuchi, "Injectable hydrogel microbeads for fluorescence-based in vivo continuous glucose monitoring," Proc. Nat. Acad. Sci. USA, vol. 107, no. 42, pp. 17 894-17 898, 2010.

[20] A. Chaudhary, M. McShane, and R. Srivastava, "Glucose response of dissolved-core alginate microspheres: Towards a continuous glucose biosensor," Analyst, vol. 135, no. 10, pp. 2620-2628, 2010.

[21] T. Förster, C. Strohhöfer, K. Bock, P. Kasak, M. Danko, Z. Kronekova, T. Nedelcev, I. Krupa, and I. Lacik, "Biosensor for calcium based on a hydrogel optical waveguide with integrated sensor proteins," in Proc. Int. Solid-State Sens., Actuat. Microsyst. Conf. (TRANSDUCERS 2009), Denver, CO, Jun., pp. 1218-1221.

[22] T. Förster, "Zwischenmolekulare Energiewanderung und Fluoreszenz,' Annalen der Physik, vol. 437, no. 1-2, pp. 55-75, 1948.

[23] ICNIRP Guidelines, "Guidelines for limiting exposure to time-varying electric, magnetic, and electromagnetic fields (up to $300 \mathrm{GHz}$ )," Health Phys., vol. 74, pp. 494-522, Apr. 1998.

[24] Wireless medium access control (MAC) and physical layer (PHY) specification for low-rate wireless personal area networks (LR-WPANs)," IEEE Standard 802.15.4, 2003.

[25] P. Valdastri, S. Rossi, A. Menciassi, V. Lionetti, F. Bernini, F. Recchia, and P. Dario, "An implantable zigbee ready telemetric platform for in vivo monitoring of physiological parameters," Sens. Actuat., A: Phys., vol. 142, no. 1, pp. 369-378, 2008.

[26] P. Valdastri, A. Menciassi, and P. Dario, "Transmission power requirements for novel zigbee implants in the gastrointestinal tract," IEEE Trans. Biomed. Eng., vol. 55, no. 6, pp. 1705-1710, Jun. 2008.

[27] ZigBee Alliance. (2009). The ZigBee alliance website [Online]. Available: http://www.zigbee.org

[28] N. R. Subramanian and N. Kirkeby, Anaren 0404 balun optimized for Texas Instruments CC2430 Transceiver, Aug. 31, 2007.

[29] K. Ye and J. S. Schultz, "Genetic engineering of an allosterically based glucose indicator protein for continuous glucose monitoring by fluorescence resonance energy transfer," Anal. Chem., vol. 75, pp. 3451-3459, 2003.

[30] W. Frommer, M. Davidson, and R. Campbell, "Genetically encoded biosensors based on engineered fluorescent proteins," Chem. Soc. Rev., vol. 38, pp. 2833-2841, 2009.

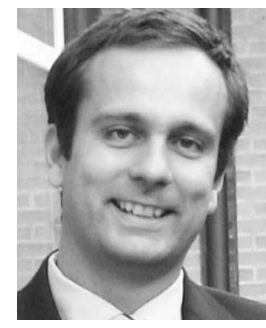

sule endoscopy.
Pietro Valdastri (M'05) received the Master's (Hons.) degree in electronic engineering from the University of Pisa, Italy, in 2002, and the Ph.D. degree in bioengineering from the Scuola Superiore Sant'Anna, Pisa, Italy, in 2006.

$\mathrm{He}$ is working on several European projects regarding the development of minimally invasive and wireless biomedical devices. He is currently an Assistant Professor of Biomedical Robotics at the Scuola Superiore Sant'Anna, where he is involved in research on implantable robotic systems and active cap-

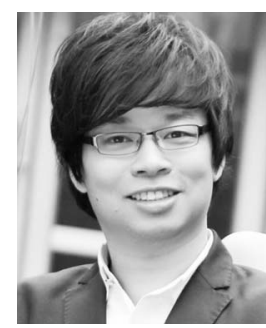

Ekawahyu Susilo received the Bachelor's degree in Electrical Engineering from Universitas Surabaya, Indonesia, in July 2000, and the Ph.D. degree in bioengineering from the Scuola Superiore Sant'Anna, Pisa, Italy, in 2009. His major is in wireless real time embedded system design

He is currently with Decawave, Ltd., Dublin Company, Fingal, Ireland, developing PHY/MAC and network layer of the new extension of IEEE 802.15.4a device using ultra wide band for better precision in 3 -D real time location system. 


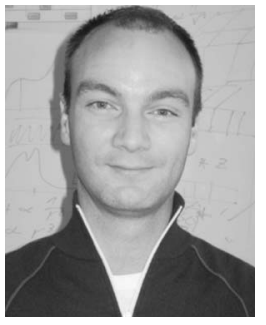

Thilo Förster received the Master's degree in physics from the Technical University of Munich, Munich, Germany, in 2005, and the Ph.D. degree in electrical engineering from the Technical University of Berlin, Berlin, Germany, in 2010.

From 2006 to 2010, he was with the Fraunhofer Research Institution for Modular Solid State Technologies Fraunhofer-Einrichtung für Modulare Festkörper-Technologien (EMFT), Munich, on several national and international research projects in the biomedical field regarding the development of biosensor systems. Since 2011, he has been a Technology Scout at Ascenion $\mathrm{GmbH}$ an intellectual property asset management company.

Christof Strohhöfer received the Master's degree in physics (Diplom-Physiker) from the University of Karlsruhe, Germany, in 1997, and the Ph.D. degree in physics from Utrecht University, The Netherlands, for work performed at the FOM-Institute for Atomic and Molecular Physics, in 2001.

In 2002, he joined Fraunhofer IZM, Munich, which became the independent Fraunhofer research institution EMFT in July 2010. In his capacity as a Senior Scientist and Project Manager, he was involved in national and European projects in the field of biosensors, biosensor fabrication technology as well as polymer technology. In May 2011, he joined BBraun Avitum AG as a Manager of New Technologies.

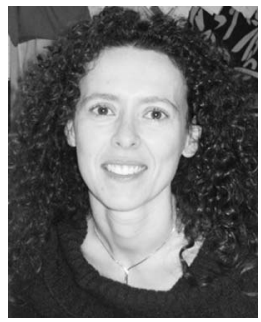

Arianna Menciassi (M'00) received the Master's degree in physics from the University of Pisa, Pisa, Italy, in 1995, and the Ph.D. degree in Biomedical Engineering from the Scuola Superiore Sant'Anna, Pisa, in 1999.

She is currently an Associate Professor of Biomedical Robotics at the Scuola Superiore Sant'Anna. Her current research interests include biomedical microrobotics for the development of innovative devices for surgery, therapy, and diagnostics.

Dr. Menciassi serves as a Co-Chair in the IEEERAS Technical Committee of Surgical Robotics and in the Editorial Board on the IEEE/ASME TRANSACTIONS ON MECHATRONICS.

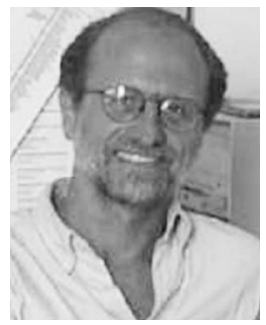

microendoscopy.

Prof. Dario is a recipient of the Joseph Engelberger Award as a Pioneer of Biomedical Robotics.

Paolo Dario (F'02) received the Master's degree in Mechanical Engineering from the University of Pisa, Pisa, Italy, in 1977.

He is a Professor of Biomedical Robotics at the Scuola Superiore Sant'Anna, Pisa, where he supervises a team of about 150 young researchers. He is the author of more than 160 ISI journal papers, many international patents, and several book chapters on medical robotics. His main research interests include biorobotics, including mechatronic and robotic systems for rehabilitation, prosthetics, surgery, and 